\title{
Paroxysmal pain during spinal anesthesia
}

\author{
Cheon-Hee Park ${ }^{1}$, Hong Chan Park ${ }^{1}$, Yong Seok Lim ${ }^{1}$, Dae Il Park ${ }^{1}$, and Hyung Jin Kim² \\ Department of Anesthesiology and Pain Medicine, ${ }^{1}$ Kwangju Christian Hospital , ${ }^{2}$ Chosun University Hospital, Gwangju, Korea
}

A 69-year-old female of American Society of Anesthesiologists physical status II was admitted for a left total knee replacement. She had injured her left knee with a crump when she had picked up something to her right side 2 months prior. She complained of a limping gait due to a disturbance in walking, with a score of 20 on the visual analog scale (VAS) ranging from 0 to 100 ( 0 , no pain; 100 , worst pain imaginable). She had had a history of hypertension for over 10 years. The laboratory data showed within-normal ranges, except for the following: VDRL 1: 1 reactive and TPHA 1: 160++ reactive. She had been treated for syphilis 30 years prior, and her symptoms and signs were clear. The electrocardiography (ECG) and chest X-ray showed nonspecific findings.

No premedication was used. Upon the patient's arrival in the operating room, standard monitors including an ECG, pulse oximeter, and noninvasive blood pressure device were installed. Spinal anesthesia was performed in the left decubitus position. $11 \mathrm{mg}$ of $0.5 \%$ hyperbaric bupivacaine were injected. The loss of sensation to cold, as tested, was up to the T10 level. Ten minutes after the spinal anesthesia, the patient complained of a lightning and clenching pain in her entire left leg. The pain intensity was up to 90 on the VAS. She received midazolam $2 \mathrm{mg}$ intravenously (IV) and dexmedetomidine $0.4 \mu \mathrm{g} / \mathrm{kg} / \mathrm{hr}$ IV. She became sedated and we believed that the pain had been relieved. However, when she awoke 10 minutes after the surgical incision, 40 minutes after the spinal anesthesia, she complained of a second pain similar to the previous one in the same lesion. She could not see her own legs as they were covered by a sterilized cloth for the operation. Propofol $30 \mathrm{mg}$ IV was immediately administered and she became sedated again. Dexmedetomidine $0.7 \mu \mathrm{g} / \mathrm{kg} / \mathrm{hr}$ IV was administered continuously so that she remained sedated.
During the operation, the surgeon changed the plan from a total knee replacement to a synovectomy due to the severe inflammation of the knee. The operation lasted 80 minutes. After applying the dressing at the operation site, the dexmedetomidine infusion was discontinued. When the patient awakened, she could see her own legs and was informed that the operation was finished. The pain had completely disappeared and did not recur. At that time, the sensory block level was T12. After the action of the bupivacaine had entirely disappeared, no sign of spinal cord irritation was found. The postoperative period was unremarkable.

The patient was rescheduled for the total knee replacement surgery one week later. With midazolam $2 \mathrm{mg}$ intramuscularly as premedication, we again performed the spinal anesthesia with a mixture of $0.5 \%$ hyperbaric bupivacaine $12 \mathrm{mg}$ and fentanyl $15 \mu \mathrm{g}$, which the patient complied with. The loss of sensation to cold, as tested, was up to the T9 level. We immediately applied midazolam $2 \mathrm{mg}$ IV and dexmedetomidine $0.7 \mu \mathrm{g} / \mathrm{kg} / \mathrm{hr}$ IV. She became sedated and remained so for the duration of the operation, which was uneventfully completed in 2 hours. The postoperative period was unremarkable. We did not perform additional neurological tests such as consultation of a neurologist or a CT, MRI, or cerebrospinal fluid tapping study because no particular neurological complications were found after the two anesthesias.

We experienced a case of a 69-year-old female complaining of a severe lightning and clenching pain in her entire left leg during spinal anesthesia. Instances of phantom pain during neuraxial anesthesia have been reported. However, these have been mainly in amputees; this phenomenon is unusual in non-amputees like our patient. We discuss the various mechanisms and treatments for this below.

Sudden and severe lightning pains involving many organs

Corresponding author: Cheon-Hee Park, M.D., Ph.D., Department of Anesthesiology and Pain Medicine, Kwangju Christian Hospital, 37, Yangnim-ro, Nam-gu, Gwangju 509-715, Korea. Tel: 82-62-650-5150, Fax: 82-62-650-5116, E-mail: pch1962@hanmail.net

(c) This is an open-access article distributed under the terms of the Creative Commons Attribution Non-Commercial License (http:// creativecommons.org/licenses/by-nc/3.0/), which permits unrestricted non-commercial use, distribution, and reproduction in any medium, provided the original work is properly cited. 
are a characteristic symptom of tabes dorsalis. Sugiyama et al. [1] reported that the lightning pains of tabes dorsalis could be triggered by spinal anesthesia. Yamashita et al. [2] reported that the patient in their study had had a positive serologic test and that $20 \%$ of the aging patients with a positive serologic test are known to have asymptomatic neurosyphilis, so that the patient had a potential for dysfunction of the dorsal roots. Although our patient was not clearly diagnosed with neurosyphilis, she had the potential for dysfunction of the dorsal roots with a positive serologic examination. We believe that one cause for the pain experienced by the patient during the spinal anesthesia might have been a dysfunction of the dorsal roots associated with neurosyphilis, in the absence of any other possible cause.

In tabes dorsalis, the degeneration involves the dorsal roots and posterior columns of the spinal cord [3]. When the ascending impulses in the posterior columns are cut off, a harmful stimulus is generated in response. This phenomenon is caused by the loss of the presynaptic inhibitory activity of the interneurons contained in the ascending nerve fibers of the dorsal column. In cases of tabes dorsalis, spinal anesthesia can therefore induce paroxysmal pain as the ascending signals in the posterior columns are cut off, causing abnormal presynaptic inhibitory activity.

Fast $\mathrm{A} \delta$ fibers and slower $\mathrm{C}$ fibers are responsible for pain transmission, and there is a modulation of the latter by the former and by nonpain sensory $A \beta$ fibers. The $C$ fibers run with the sympathetic afferents and they can ascend a variable distance within the sympathetic chain so as to enter the spinal cord high- er than the level of the associated $A \beta$ and $A \delta$ fibers, the so-called paraspinal pathway of sensory input. Blockade A fibers may reduce the inhibition of the transmission of impulses via $C$ fibers and unmask previously unfelt pain in the presence of abnormal sensory pathways or activity [4]. In relation to neurosyphilis, we believe that the dorsal roots and posterior columns could have degenerated subclinically, in both Yamashita et al.s [2] and our case.

Regarding phantom pain during regional anesthesia, Koyama et al. [5] suggested that the most effective treatment for this symptom was a subanesthetic dose of thiopental. This has been found to be more effective than morphine, fentanyl, pethidine or diazepam. This finding is particularly relevant in view of the use of subanesthetic doses of thiopental for diagnostic testing in the subclassification of deafferentation and central pain syndrome. Our patient was treated with midazolam, propofol, and dexmedetomidine. We suggest that phantom pain during regional anesthesia is treated more effectively with a sedative than with an opioid. Accordingly, our patient was treated with a sedative. At that point, we believe that her paroxysmal pain was a kind of phantom pain.

In conclusion, we witnessed a case of severe paroxysmal pain during spinal anesthesia. We discussed several possible mechanisms for the positive serologic examination of syphilis, and suggested that the most effective treatment for paroxysmal pain during regional anesthesia was the use of a sedative such as thiopental, midazolam, propofol, or dexmedetomidine, rather than an opioid.

\section{References}

1. Sugiyama D, Nobuhara K, Maekawa N, Obara H. Severe lightning pain after spinal anesthesia in a patient with tabes dorsalis. Anesth Analg 1989; 69: 268-9.

2. Yamashita S, Joukou M, Kuramoto T. Severe lightning pain during spinal anesthesia. Masui 1990; 39: 1708-10.

3. Ganong WF. Cutaneous, deep, \& visceral sensation. In: Review of Medical Physiology. 14th ed. Connecticut, Appleton \& Lange. 1989 , p 108.

4. DeJong R, Cullen SC. Theoretical aspects of pain: bizarre pain phenomena during low spinal anesthesia. Anesthesiology 1963; $24: 628-35$.

5. Koyama K, Watanabe S, Tsuneto S, Takahashi H, Naito H. Thiopental for phantom limb pain during spinal anesthesia. Anesthesiology 1988; 69: 598-600. 